\title{
Expression of Phospho-Elk-1 in Rat Gut after the Whole Body Gamma Irradiation
}

\author{
D. DRIÁK ${ }^{1,2}$, J. ÖSTERREICHER ${ }^{2}$, Z. ŘEHÁKOVÁ ${ }^{2}$, Z. VILASOVÁ ${ }^{2}$ J. VÁVROVÁ \\ ${ }^{1}$ Gynecology and Obstetrics Clinic, First Faculty of Medicine, Charles University and University \\ Hospital Bulovka, Prague, Czech Republic and ${ }^{2}$ Department of Radiobiology and Molecular \\ Pathology, Faculty of Military Health Sciences, University of Defense, Hradec Králové, Czech \\ Republic
}

Received May 16, 2006

Accepted July 17, 2007

On-line October 11, 2007

\begin{abstract}
Summary
Gastrointestinal form is the second stage of acute radiation syndrome (ARS) with a threshold dose of 8 Gy in man. It represents an absolutely lethal clinical-pathological unit, necrohemorrhagic enteritis and proctocolitis, with unknown causal therapy. Elk-1 is a protein acting as a transcription factor activating specified genes. The purpose of our study was to examine the expression of phospho-Elk-1 in irradiated jejunum and transversal colon of rats with radiation-induced enterocolitis and to assess the importance of this transcriptional factor as a biodosimetric marker of radiation-induced enteropathy. The laboratory rats were randomly divided into 21 groups, 10 animals per group, and irradiated with whole body $y$-irradiation of 1,5 , 10,15 , and $20 \mathrm{~Gy}$. Samples of jejunum and transversal colon were taken 24, 48, 72, and 96 hours later, immunohistochemically stained, and the phospho-Elk-1 expression was examined using computer image analysis. A group of 10 shamirradiated animals was used as control. Significantly increased expression of phospho-Elk-1 in rat jejunum has been found in all time intervals after irradiation by sublethal doses of 1 and $5 \mathrm{~Gy}$, whereas after the irradiation by lethal doses, the expression of phospho-Elk-1 in rat jejunum varied considerably. Significantly increased expression of phospho-Elk-1 in transversal colon has also been found in the first days after irradiation by sublethal doses of 1 and $5 \mathrm{~Gy}$. After irradiation by lethal doses, tere was no uniform pattern of the changes in the expression of phospho-Elk1 in rat transversal colon. The detection of phospho-Elk-1 might be considered as a suitable and very sensitive biodosimetric marker of radiation-induced injury of small and large intestine. According to our knowledge, this is the first study on the phospho-Elk-1 expression in irradiated jejunum and transversal colon in the rat.
\end{abstract}

\section{Key words}

Radiation-induced entero-colitis • Phospho-Elk-1 expression • Biodosimetric marker

\begin{abstract}
Corresponding author
Daniel Driák, Gynecology Obstetrics Clinic, University Hospital Bulovka, Budínova 2, 180 81, Prague 8, Czech Republic. Fax: +420 28384 0507. E-mail: driak@seznam.cz
\end{abstract}

\section{Introduction}

Radiation-induced enterocolitis is a form of acute radiation disease developing after the absorption of dose of 8 Gy and higher. It concerns an absolutely lethal clinical-pathological unit, necro-haemorrhagic enteritis and colitis, for which no causal therapy is known (Fajardo 1982, Nguyen et al. 2002, Österreicher et al. 2003). The large morphological changes in small intestine mucosa develop rapidly and progressively during the first 4 days after irradiation with supralethal doses.

Molecular biology explains the effect of irradiation on cells via the alteration of signaling pathways (Stulík et al. 2000, Suzuki et al. 2001, Torii et al. 2004). Elk-1 is a protein acting as a transcription factor inducing c-fos transcription and activating specified genes of DNA. Elk-1 is a component of the ternary complex that binds the serum response element (SRE) in response to serum and growth factor. Afterwards, Elk-1 C-terminal region at multiple sites is phosphorylated by mitogen-activated protein kinases (MAPK, extracellular signal-related kinases ERK1 and ERK2), particularly phosphorylation on site of $\mathrm{Ser}^{383}$ is critical for transcriptional activation. Elk-1 appears to be a direct target of activated MAPK pathways, besides, the 
locus of $\operatorname{Ser}^{383}$ is also a target of the stress-activated kinases (SAPK/JNK1, JNK2 and p38) (Cavigelli et al. 1995, Kasza et al. 2005, Marais et al. 1993, Murai et al. 2002, Narang and Krishna 2004, Salinas et al. 2004, Whitmarsh et al. 1995). Phosphorylation of Elk-1 by ERK2 is a major link in the Raf-1 kinase-dependent signal transduction pathway that activates c-fos expression (Kortenjann et al. 1994). The Elk-1 transcription factor integrates MAPK signaling pathways in vivo to co-ordinate biological response to different extracellular stimuli (Whitmarsh et al. 1995).

In this study, we examined the expression of Elk-1 phosphorylated on $\mathrm{Ser}^{383}$ in rat jejunum and transversal colon and its dependence on dose and time after the whole body $\gamma$-irradiation.

\section{Methods}

Randomly divided into 21 groups (10 animals per group), male Wistar rats (Konárovice, Czech Republic) aged 12-16 weeks and weighing 250-300 g were given the whole body $\gamma$-irradiation using a ${ }^{60} \mathrm{Co}$ unit (Chisotron Chirana, Czech Republic) at a dose rate of $1.0 \mathrm{~Gy} / \mathrm{min}$ at a distance of $100 \mathrm{~cm}$ from the skin and at doses of 1, 5, 10, 15, and 20 Gy. The animals were lightly anesthetized before irradiation or sham-irradiation using a mixture of one volume of Rometar (Spofa Company, Prague, Czech Republic), three volumes of Narkamon (Léčiva Company, Prague, Czech Republic) and 12 volumes of physiological saline. This solution was injected intramuscularly at $1.0 \mathrm{ml} / \mathrm{kg}$. After 24, 48, 72, and 96 hours, the animals were sacrificed by a cervical dislocation. The rats of a sham-irradiated group were killed after 72 hours. Only one animal of each group survived 96 hours after irradiation with doses of 15 and 20 Gy. These groups were omitted from the statistical analysis. All the procedures involving animals were approved by the Ethics Committee of Faculty of Military Health Sciences in Hradec Králové.

\section{Histological examination}

Samples were taken from the proximal part of jejunum (approximately $2 \mathrm{~cm}$ aborally from duodenojejunal flexure) and of the middle part of transversal colon. One transversal section of both jejunum and transversal colon per animal was examined. All the samples were incubated in $10 \%$ formalin-buffered solution and fixated up to 24 hours. The immunohistochemical detection of phospho-Elk-1 was performed on epithelial cells with a standard immunoperoxidase technique. After blocking the endogenous peroxidase activity for $20 \mathrm{~min}$, the tissue sections were incubated for 1 hour with the biotinylated mouse monoclonal antibody specifically recognizing Elk-1 phosphorylated on $\mathrm{Ser}^{383}$ (Cell Signaling, USA) diluted 1:200 in phosphated buffer saline (PBS, pH 7.2). Afterwards, the sections were washed three times in PBS. All slides were then incubated for $20 \mathrm{~min}$ with a secondary antimouse antibody under the same condition. Excess antibodies were washed off with PBS. All slides were incubated with an enzymatic complex streptavidin-peroxidase (Universal large volume DAKO LSAB + Kit, Peroxidase - DAKO Corporation, Carpinteria, USA) and washed with PBS.

Finally, $0.05 \%$ 3,3-diaminobenzidinetetrahydrochloride-chromogen solution (Sigma, St. Louis, MO, USA) in PBS containing $0.02 \%$ hydrogen peroxide was added for 20 minutes to visualize the antigen-antibody complex in situ.

\section{Measurement of phospho-Elk-1 expression}

Stained samples were evaluated using a BX 51 microscope (Olympus Company, Prague, Czech Republic) and a computer image analysis ImagePro 4.11 (Media Cybernetics, USA). Immunoreactive structures in epithelium were detected in the ranges red 100-255, green 161-255, and blue 171-255. Six viewing microscopic fields at a 400-fold original magnification were randomly selected of each jejunum and colon sample so that 60 measurements were performed per group. To increase the validity of results, we have used three parameters of the computer image analysis for quantification of the phospho-Elk-1 expression: sum of density mean, density per area (percentual positivity per microscopic field) and integral optic density (IOD).

\section{Data processing}

The Mann-Whitney rank sum test was used for the statistical analysis. Significance level was set at $\mathrm{p}<0.05$. Data are given as arithmetic mean \pm S.E.M.

\section{Results}

Sum of density mean of phospho-Elk-1 positive cells of rat jejunum (Fig. 1)

The value of sum of density mean is significantly higher in rats after irradiation by doses of 1 and 5 Gy in all time intervals with the maximum 


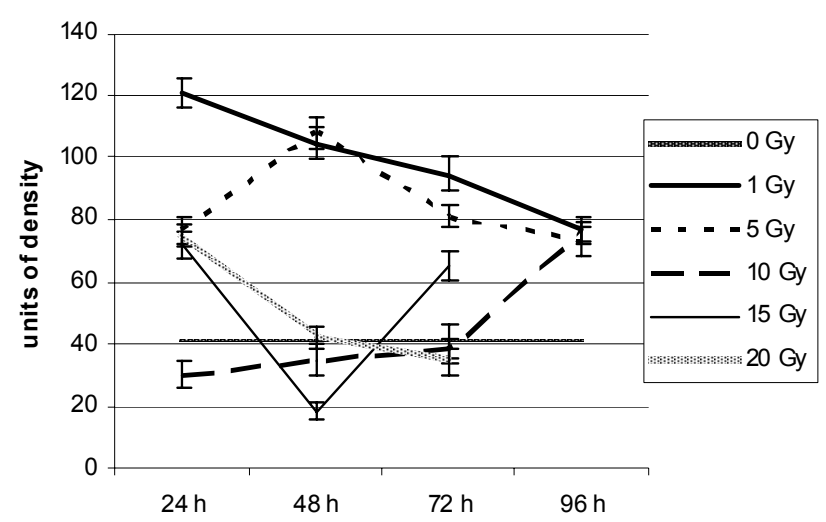

Fig. 1. Sum of density mean of p-Elk-1 positive cells in irradiated rat jejunum in arithmetic mean \pm S.E.M.

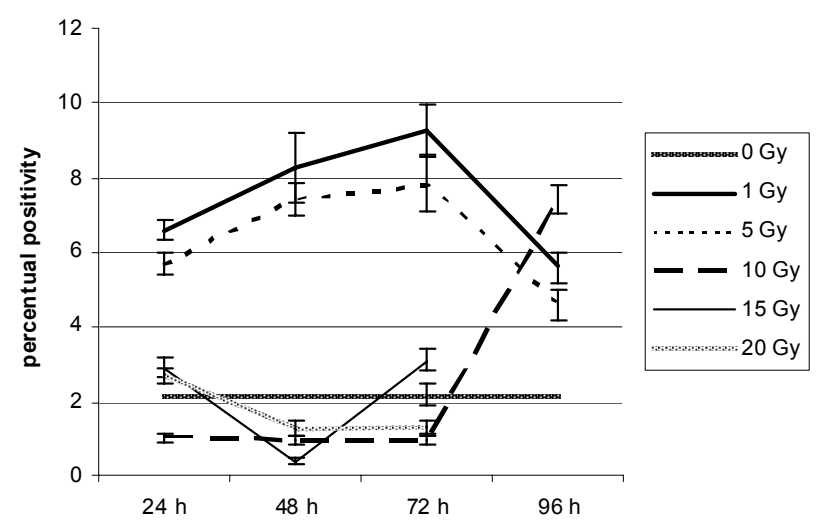

Fig. 2. Per area density of p-Elk-1 positive cells in irradiated rat jejunum in arithmetic mean \pm S.E.M.

24 hours after irradiation by dose of $1 \mathrm{~Gy}$. Significantly higher values of sum of density mean were found in rats 96 hours after irradiation by dose of $10 \mathrm{~Gy}, 24$ and 72 hours after irradiation by dose of $15 \mathrm{~Gy}$, and 24 hours after irradiation by dose of 20 Gy. Significantly lower value was found in rats 48 hours after irradiation by dose of $15 \mathrm{~Gy}$.

Per area density of phospho-Elk-1 positive cells of rat jejunum (Fig. 2)

The value of per area density is significantly higher in rats after irradiation by doses of 1 and $5 \mathrm{~Gy}$ in all time intervals with the maximum 72 hours after irradiation by dose of $1 \mathrm{~Gy}$. Significantly higher values of per area density were found in rats 96 hours after irradiation by dose of $10 \mathrm{~Gy}$. Significantly lower value was found in rats 24-72 hours after irradiation by dose of $10 \mathrm{~Gy}$, and 48 hours after irradiation by dose of $15 \mathrm{~Gy}$.

Integral optic density of phospho-Elk-1 positive cells of

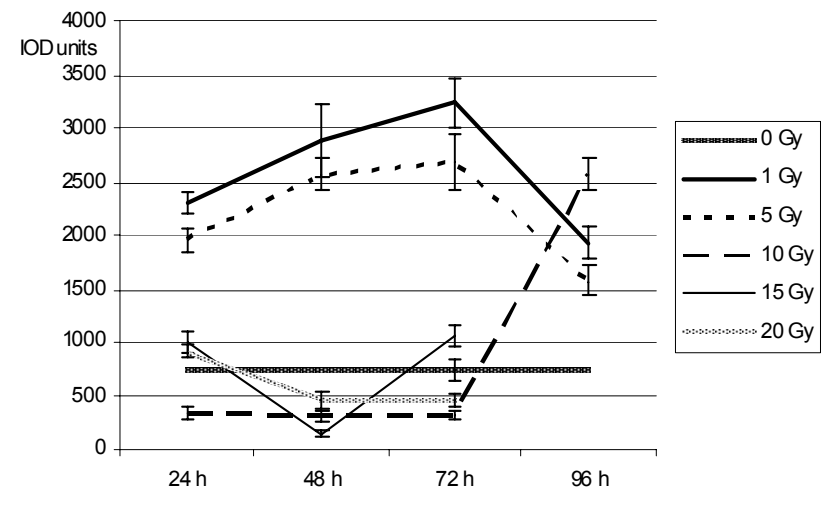

Fig. 3. Integral optic density of p-Elk-1 positive cells in irradiated rat jejunum in arithmetic mean \pm S.E.M.

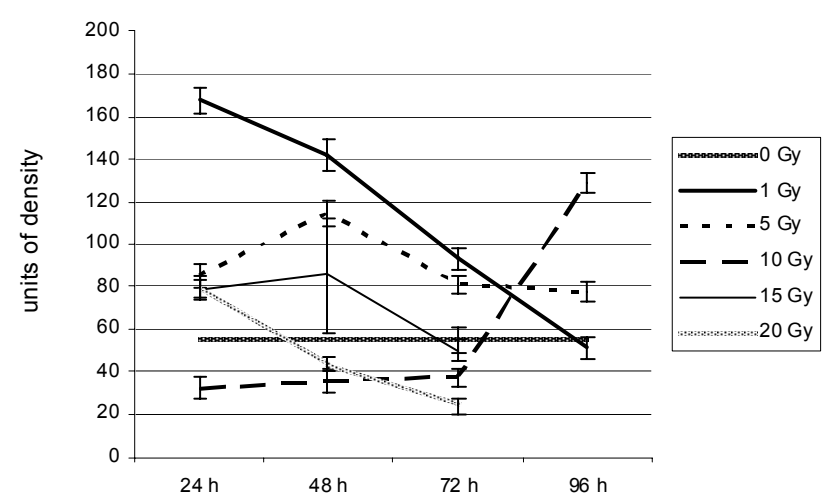

Fig. 4. Sum of density mean of p-Elk-1 positive cells in irradiated rat colon in arithmetic mean \pm S.E.M.

rat jejunum (Fig. 3)

The value of integral optic density is significantly higher in rats after irradiation by doses of 1 and 5 Gy in all time intervals with the maximum 72 hours after irradiation by dose of 1 Gy. Significantly higher values of integral optic density were found in rats 96 hours after irradiation by dose of $10 \mathrm{~Gy}$. Significantly lower value was found in rats 24-72 hours after irradiation by dose of $10 \mathrm{~Gy}$, and 48 hours after irradiation by dose of $15 \mathrm{~Gy}$.

Sum of density mean of phospho-Elk-1 positive cells of rat transversal colon (Fig. 4)

The value of sum of density mean is significantly higher in rats 24-72 hours after irradiation by dose of 1 Gy with the maximum 24 hours after irradiation. Significantly higher values of sum of density mean were found in rats irradiated by dose of 5 Gy in all time intervals, 96 hours after irradiation by dose of $10 \mathrm{~Gy}, 24-48$ hours after irradiation by dose of $15 \mathrm{~Gy}$, 


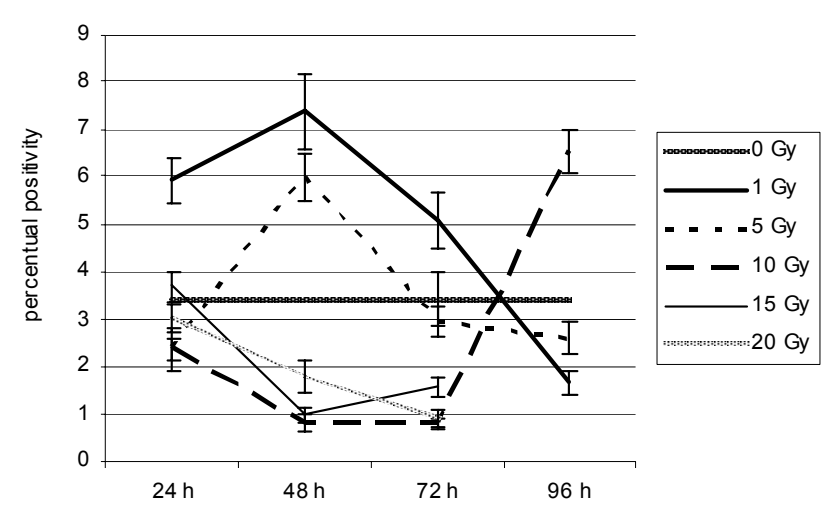

Fig. 5. Per area density of p-Elk-1 positive cells in irradiated rat colon in arithmetic mean \pm S.E.M.

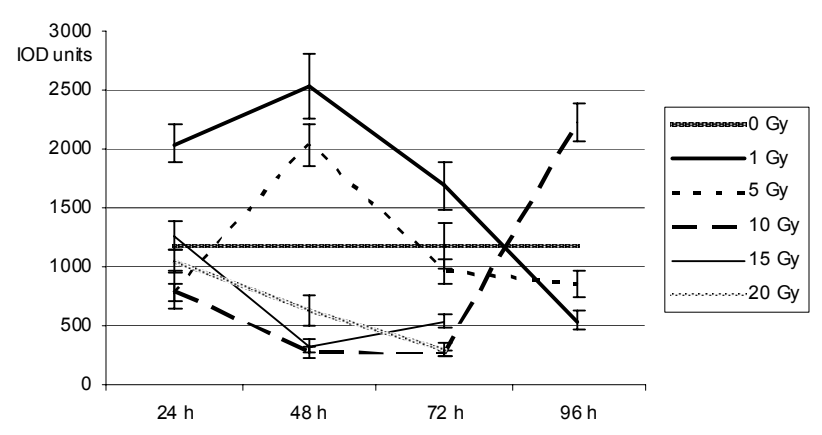

Fig. 6. Integral optic density of $\mathrm{p}$-Elk-1 positive cells in irradiated rat colon in arithmetic mean \pm S.E.M.

and 24 hours after irradiation by dose of 20 Gy. Significantly lower value was found in rats 24-72 hours after irradiation by dose of $10 \mathrm{~Gy}$, and 72 hours after irradiation by dose of $20 \mathrm{~Gy}$.

Per area density of phospho-Elk-1 positive cells of rat transversal colon (Fig. 5)

The value of per area density is significantly higher in rats 24-72 hours after irradiation by dose of 1 Gy with the maximum 48 hours after irradiation. Significantly higher values of per area density were found in rats 48 hours after irradiation by dose of $5 \mathrm{~Gy}$, and 96 hours after irradiation by dose of 10 Gy. Significantly lower values were found in rats 96 hours after irradiation by dose of $1 \mathrm{~Gy}, 48-72$ hours after irradiation by dose of $10 \mathrm{~Gy}, 48-72$ hours after irradiation by dose of $15 \mathrm{~Gy}$, and 72 hours after irradiation by dose of $20 \mathrm{~Gy}$.

Integral optic density of phospho-Elk-1 positive cells of rat transversal colon (Fig. 6)

The value of integral optic density is significantly higher in rats $24-72$ hours after irradiation by dose of 1 Gy with the maximum 48 hours after irradiation. Significantly higher values of integral optic density were found in rats 48 hours after irradiation by dose of $5 \mathrm{~Gy}$, and 96 hours after irradiation by dose of 10 Gy. Significantly lower values were found in rats 96 hours after irradiation by dose of 1 Gy, 48-72 hours after irradiation by dose of $10 \mathrm{~Gy}$, and 72 hours after irradiation by dose of $20 \mathrm{~Gy}$.

\section{Discussion}

Ionizing radiation activates the typically mitogen-activated pathway (MAPK/ERK cascade) along with the activation of stress-responsing pathways, NF- $\mathrm{B}$, and many immediate early genes as c-jun, c-fos, and egr-1. The original papers concerning signaling pathways alteration in enterocytes are rather scarce. Description of gene expression pattern in colonic crypts of adenomas with low grade dysplasia has shown that the activation of the MAPK pathway seems to be an early sign of carcinogenesis (Lechner et al. 2003). The works studying molecular changes in gastrointestinal tract after exposure to ionizing radiation are even more rare. Österreicher et al. (2007) search for new and sensitive biodosimetric markers of radiation-induced enterocolitis.

Recent paper of Narang and Krishna (2004) describes the MAPK signaling pathway changes in rat liver. Ionizing radiation activates both the cytoprotective mitogen-activated protein kinases (MAPK, extracellular signal-related kinases ERK1, ERK2) and the cytotoxic stress-activated kinases (SAPK/JNK1, JNK2, p38). Each of them sends divergent signals to the nucleus. There is a chronological order of activation of the kinases and a dose-dependent activation. The cytoprotective ERK2 shows a progressive increase in expression with dose and a prolonged response to stimuli, whilst the cytotoxic SAPK is activated by very low doses of ionizing radiation $(0.1 \mathrm{~Gy})$ at early period. The duration of activation of kinases seems to be the decisive factor of whether the cell goes into proliferation and differentiation or cell-cycle arrest. A transient activation of ERK leads to proliferation, whereas persistent activation leads to cellcycle $\mathrm{G}_{2} / \mathrm{M}$ arrest, and apoptosis is possible. Low doses of ionizing radiation ( $1 \mathrm{~Gy}$ ) cause a prolonged activation of MAPK and SAPK. Higher doses of radiation (6 Gy) cause a much weaker activation of the MAPK cascade, but a similar degree of SAPK activation (Narang and Krishna 2004).

Transcription factor Elk-1 is a common target of activated MAPK pathways and the SAPK pathways, the 


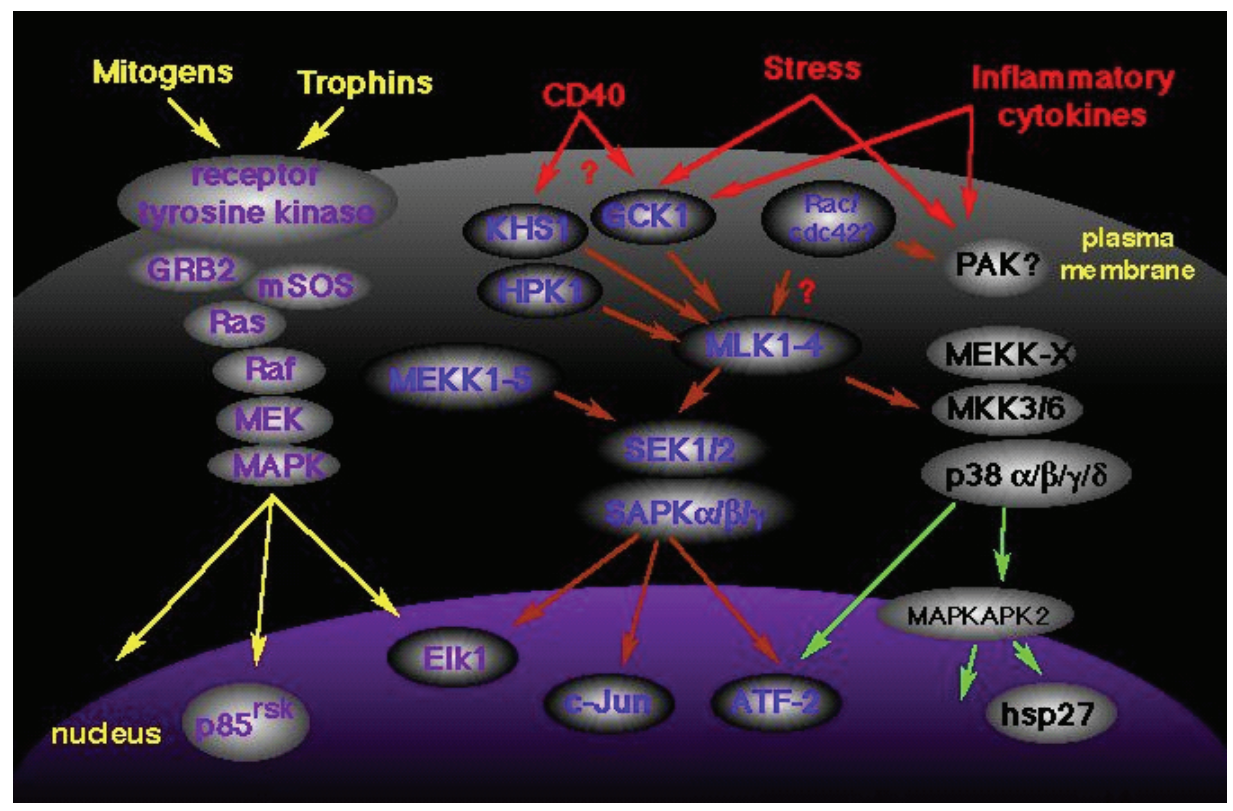

Fig. 7. Signaling map of three kinases pathways

two signaling pathways converge by means of Elk-1 (Fig. 7). The expression of Elk in response to dose correlates very well with the activation of ERK2 at higher doses (1-5 Gy) in rat liver (Narang and Krishna 2004). The expression of the precursor to SAPK/JNK was induced at a lower dose with maximum at the dose of 0.5 Gy and declined at the dose of 1 Gy. A complete inhibition of induction of expression occurred at the doses of 2-5 Gy. The expression of phospho-Elk did not increase at the doses of 0.1-0.5 Gy. According to the study on irradiated rat liver, ionizing radiation stimulates both the ERK and the JNK pathway to phosphorylation of Elk-1, but in a different chronological order and in a different dose-dependence (Narang and Krishna 2004).

According to our knowledge, this is the first study detecting and quantifying the phospho-Elk-1 expression in irradiated rat gut. We observed a significantly increased expression of phospho-Elk-1 in rat jejunum in all time intervals after irradiation by sublethal doses of 1 and $5 \mathrm{~Gy}$. After irradiation by lethal doses, the expression of phospho-Elk-1 in rat jejunum was significantly both increased and decreased, or did not indicate significant changes.

We also observed a significantly increased expression of phospho-Elk-1 in rat transversal colon in the first days after irradiation by sublethal doses of 1 and 5 Gy. After irradiation by lethal doses, the expression of phospho-Elk-1 in rat transversal colon was again highly variable. Although epithelial denudation after lethal irradiation is usually described, our time-dose intervals were focused to clinically prodromal and latent phases when lower number of enterocytes is compensated by their flattening. Therefore, we had the opportunity to measure the phospho-Elk-1 expression in sufficient number of enterocytes. Only one animal of each group survived 96 hours after irradiation by doses of 15 and 20 Gy, where large devastating effects and remarkable loss of enterocytes were observed. Due to the low amount of epithelial cells, these groups were omitted from the statistical analysis.

The variance of findings after irradiation by lethal doses can be explained with a different velocity of signaling pathway changes after irradiation by various doses. The other explanation might be the exhaustion of produced signaling molecules in injured cells. The increased values 96 hours after irradiation by dose of 10 Gy may be due to the resynthesis of signaling molecules. After irradiation by doses higher than $10 \mathrm{~Gy}$, the resynthesis is not sufficient. Our hypotheses have to be verified by further experiments investigating the phospho-Elk-1 expression in the first hours after irradiation (1, 2 and 4 hours). In the study on irradiated rat liver, the expression of the signaling factors was examined at 4 hours after irradiation (Narang and Krishna 2004).

In comparison with radiation-induced enteritis, increased values of phospho-Elk-1 expression in transversal colon have not been observed 96 hours after irradiation with dose of $1 \mathrm{~Gy}$ and in several time intervals after irradiation with dose of 5 Gy. This might be explained by a rather different (slower) cytokinetics and repopulation of large intestine mucosa and earlier exhaustion of signaling molecules. After irradiation by lethal doses the dynamics of changes in both jejunum and 
transversal colon are very similar.

Based on the results of our study, we suppose that phospho-Elk-1 is a very sensitive parameter of radiation-induced gut injury, significantly elevated after irradiation by sublethal doses. The detection of phosphoElk-1 might be considered as a suitable biodosimetric marker useful under clinical conditions, and possibly in military field conditions. However, this suggestion has to be verified by further experiments detecting the phosphoElk-1 expression in other tissues and after other nuclear, biological and chemical agents than ionizing radiation.

Besides radiation, the phosphorylation of Elk-1 is stimulated by a wide variety of extracellular stimuli, e.g. visible or UV light (Price et al. 1996, Coogan and Piggins 2003, Kaminska et al. 1999). Besides the oxidative stress, other stressors could induce the phosphorylation of Elk-1. Receptor-bound growth and neurotrophic factors induce Elk-1 phosphorylation and c-fos transcription through ERK activation, UV irradiation stimulates Elk-1 activity through JNK activation (Cavigelli et al. 1995). In such case, it might be a universal response to the cellular stress caused by an extracellular signal. However, there will be probably a different order of time- and dose-dependence of activation.

\section{Conclusions}

Significantly increased expression of phospho-
Elk-1 in rat jejunum in all time intervals, i.e. 24, 48, 72 , and 96 hours after irradiation by sublethal doses of 1 and 5 Gy has been observed. We can suppose that, at this time, phospho-Elk-1 is the most sensitive biodosimetric marker of radiation-induced enteropathy. After irradiation by lethal doses, the expression of phospho-Elk-1 in rat jejunum was highly variable. Similarly, increased expression of phospho-Elk-1 in rat transversal colon in the first days after irradiation by sublethal doses of 1 and 5 Gy have been observed, whereas after irradiation by lethal doses, the expression of phospho-Elk-1 in rat colon transversum was significantly increased, decreased, or did not change. The increase of phospho-Elk-1 expression was higher in irradiated jejunum than in transversal colon, that confirms the suggestion that the small intestine is more radiosensitive than the large one.

The detection of phospho-Elk-1 might be considered as a suitable and sensitive biodosimetric marker of radiation-induced injury of small and large intestine. Further experiments verifying the expression of phospho-Elk-1 in other tissues are needed. We propose further testing of other agents, in addition to the ionizing radiation. If our results will be confirmed, phospho-Elk-1 might be supposed as a useful parameter under clinical conditions, and possibly in military field conditions.

\section{Conflict of Interest}

There is no conflict of interest.

\section{References}

CAVIGELLI M, DOLFI F, CLARET FX, KARIN M: Induction of c-fos expression through JNK-mediated TCF/Elk-1 phosphorylation. EMBO J 14: 5957-5964, 1995.

COOGAN AN, PIGGINS HD: Circadian and photic regulation of phosphorylation of ERK1/2 and Elk-1 in the suprachiasmatic nuclei of the Syrian hamster. J Neurosci 23: 3085-3093, 2003.

FAJARDO LF: Pathology of Radiation Injury. Masson Publishing, New York, 1982.

KAMINSKA B, KACZMAREK L, ZANGENEHPOUR S, CHAUDHURI A: Rapid phosphorylation of Elk-1 transcription factor and activation of MAP kinase signal transduction pathways in response to visual stimulation. Mol Cell Neurosci 13: 405-414, 1999.

KASZA A, O'DONNELL A, GASCOIGNE K, ZEEF LA, HAYES, A, SHARROCKS AD: The ETS domain transcription factor elk-1 regulates the expression of its partner protein, SRF. $J$ Biol Chem 280: 1149-1155, 2005.

KORTENJANN M, THOMAE O, SHAW PE: Inhibition of v-raf-dependent c-fos expression and transformation by a kinase-defective mutant of the mitogen-activated protein kinase Erk2. Mol Cell Biol 14: 4815-4824, 1994.

LECHNER S, MÜLLER-LADNER U, RENKE B, SCHÖLMERICH J, RÜSCHOFF J, KULLMANN F: Gene expression pattern of laser microdissected colonic crypts of adenomas with low grade dysplasia. Gut 52: 1148$1153,2003$. 
MARAIS R, WYNNE J, TREISMAN R: The SRF accessory protein Elk-1 contains a growth factor-regulated transcriptional activation domain. Cell 73: 381-393, 1993.

MURAI K, TREISMAN R: Interaction of serum response factor (SRF) with the Elk-1 B box inhibits RhoA-actin signaling to SRF and potentiates transcriptional activation by Elk-1. Mol Cell Biol 22: 7083-7092, 2002.

NARANG H, KRISHNA M: Mitogen-activated protein kinases: specifity of response to dose of ionizing radiation in liver. J Radiat Res 45: 213-220, 2004.

NGUYEN NP, ANTOINE JE, DUTTA S, KARLSSON U, SALLAH, S: Current concepts in radiation enteritis and implications for future clinical trials. Cancer 95: 1151-1163, 2002.

ÖSTERREICHER J, VÁVROVÁ J: Lectures in Radiobiology. (in Czech) Manus, Prague, 2003.

ÖSTERREICHER J, DRIÁK D, VILASOVÁ Z, VÁVROVÁ J: Impact of phospho-Elk-1 expression in enterocytes in biological dosimetry after low-dose irradiation. Def Sci J 57: 845-851, 2007.

PRICE MA, CRUZALEGUI FH, TREISMAN R: The p38 and ERK MAP kinase pathways cooperate to activate ternary complex factors and c-fos transcription in response to UV light. EMBO J 15: 6552-6563, 1996.

SALINAS S, BRIANCON-MARJOLLET A, BOSSIS G, LOPEZ MA, PIECHACZYK M, JARIEL-ENCONTRE I, DEBANT A, HIPSKIND RA: SUMOylation regulates nucleo-cytoplasmic shuttling of Elk-1. J Cell Biol 165: 767-773, 2004.

STULÍK J, ÖSTERREICHER J, KAFFENBERGER W, MACELA A: Radiation-induced signaling mechanisms in cells (in Czech). Voj zdrav listy 69: 263-266, 2000.

SUZUKI K, KODAMA S, WATANABE M: Extremely low-dose ionizing radiation causes activation of mitogenactivated protein kinase pathway and enhances proliferation of normal human diploid cells. Cancer Res 61: 5396-5401, 2001.

TORII S, KUSAKABE M, YAMAMOTO T, MAEKAWA H, NISHIDA E: Sef is a spatial regulator for Ras/MAP kinase signaling. Dev Cell 7: 33-44, 2004.

WHITMARSH AJ, SHORE P, SHARROCKS AD, DAVIS RJ: Integration of MAP kinase signal transduction pathways at the serum response element. Science 269: 403-407, 1995. 